\title{
Pressure Based Segmentation in Volumetric Images
}

\author{
Thamer S. Alathari and Mark S. Nixon \\ School of Electronics and Computer Science \\ University of Southampton, SO17 1BJ, UK \\ \{tsa1g11, msn\}@ecs.soton.ac.uk
}

\begin{abstract}
Analysing Roman coins found in archaeology sites has been traditionally done manually by an operator using volumetric image slices provided by a computed tomography scanner. In order to automate the counting process, a good segmentation for the coins has to be achieved to separate the touching surfaces of the coins. Separating touching surfaces in volumetric images has not yet attracted much attention. In this paper we propose a new method based on using a form of pressure to separate the intersecting surfaces. We analogise the background of the image to be filled with an ideal gas. The pressure at a point has an inverse relationship with the volume of homogeneous material surrounding it. By studying the pressure space, the locations of intersecting surfaces are highlighted and encouraging segmentation results are achieved. Our analysis concerns a selection of images, naturally demonstrating success, together with an analysis of the new technique's sensitivity to noise.
\end{abstract}

Keywords: Physical analogy, Image segmentation, Pressure, Image thresholding, Object separation.

\section{Introduction}

Computed tomography imaging is an increasingly popular source for information about three dimensional objects, with many applications ranging from medical to industrial. Scans can contain multiple objects with the same density or single objects containing smaller ones with similar density. The placement of the objects in the 3D space can be random and in some cases the surfaces of those objects touch which makes it difficult to separate them using conventional thresholding and segmentation techniques, motivating development of a higher level process. An example of such a problem is the CT scanned jar (Fig. 1) which contains a set of Roman coins. This set of data contains coins with similar density randomly placed with different orientations and locations within the jar. The problem associated with this particular set of data relies in the high attenuation factor for the material from which the coins are made which in turn increases the chance of touching surfaces in the volumetric image especially for the coins in the centre of the jar. Separating objects with the same density and texture is challenging due to the absence of techniques for detecting in 3D the regions of intersection between the objects, impeding the possibility of counting the coins. 


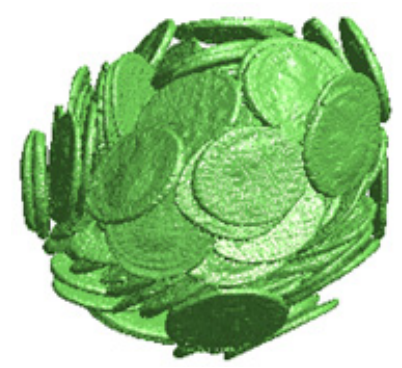

Fig. 1. CT image of Roman coins inside a jar

Many approaches have been developed to solve the problem of separating touching objects in two dimensional (2D) space. The two main application concern separating rice grains [1] and counting cells in microscope images [2]. A traditional approach involves thresholding, corner detection and joining points of interest to create a binary image of disconnected objects. On the other hand, there is no such technique for 3D image analysis. The literature provides some model-based methods that have been used to separate left and right lungs [3]. The search for regions of interest uses images where lungs intersect, to minimize the computational demands. Edge or surface detection can be applied such as a 3D Marr-Hildreth operator [4] but unfortunately those methods would not address the touching regions. Using approaches based on connectivity would consider both surfaces as a single entity. It might be possible to achieve some results by morphological analysis but such procedures are isotropic and do not adapt to local scenarios.

We present a new 3D approach based on using a physical analogy [5] to separate intersecting regions. The approach is anisotropic and does not require a model or previous knowledge about the regions of interest. It can be applied automatically so as to delineate intersecting structures by adapting locally to image content. As such the images are then rendered suitable for later analysis procedures. The analogy used is pressure, and the approach does not mimic application of pressure precisely, and more to develop a new method to separate touching objects, in $3 \mathrm{D}$, which allows for automated analysis of a Roman hoard of coins.

\section{Methods}

Given objects in a volumetric images which have the same intensity, separating them can usually be achieved by using a thresholding operation, such as Otsu [6], unless the objects surfaces are close together or touching. The area between touching objects in a CT scan image creates a very fine gradient caused by X-ray refraction exacerbating difficulty in segmenting the individual objects.

On the other hand, Otsu thresholding can remove the background well. Since the objects are solid the histogram should show a well-defined peak where the objects exist, extracting this peak would help in creating a consistent background. 
Having separated the background, the coins need to be separated. To achieve this, a physical analogy has been used to measure the local pressure in the background which has been considered to be filled with an ideal gas.

$$
P V=n R T
$$

where the number of moles $(n)$, temperature $(T)$ and ideal gas constant $R$ are constant. An inversely proportional relation between the pressure and the volume is created. To use this as an image processing operator, the pressure at each background point $\mathbf{P}_{x, y, z}$ is accumulated within a window $\mathbf{W}$ as

$$
\mathbf{P}_{x, y, z}=1 / \sum_{x, y, z \in \mathbf{W}} \mathbf{V}_{x, y, z} \quad \forall \mathbf{V}_{x, y, z} \in \text { background }
$$

where $\mathbf{V}$ is the volume of interest. The values of $\mathbf{P}$ are then thresholded to suggest where maximum pressure occurs.

$$
\mathbf{M}_{x, y, z}=\mid \begin{array}{lr}
0 & \text { if } \mathbf{P}_{x, y, z} \geq \begin{array}{r}
\text { threshold } \\
\text { otherwise }
\end{array}
\end{array}
$$

This provides a mask $\mathbf{M}$ which can be used to eliminate voxels in the original image $\mathbf{V}$. The separated image $\mathbf{S}$ is then

$$
\mathbf{S}_{x, y, z}=\mathbf{M}_{x, y, z} \times \mathbf{V}_{\mathrm{x}, \mathrm{y}, \mathrm{z}}
$$

\section{Results}

To illustrate this, the method has been applied to two touching synthetic disks (slices at different depths are shown in Fig. 2). Using Otsu thresholding revealed only a single object in the image. The pressure domain is then calculated for the disks using local pressure (Fig. 3). Based on the window size a proper threshold is applied to create a mask of the inverse of the touching area. The mask is then multiplied by the image to separate the disks (Fig. 4). The histogram of elements shows a new object which indicates a successful separation.

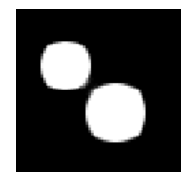

$\mathbf{V}_{x, y, 2}$

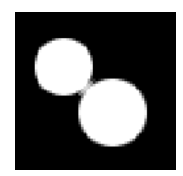

$\mathbf{V}_{x, y, 4}$

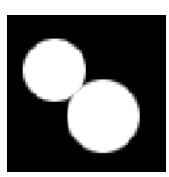

$\mathbf{V}_{x, y, 6}$

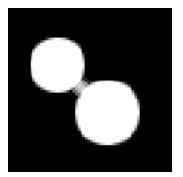

$\mathbf{V}_{x, y, 8}$

Fig. 2. Different depth slices of two touching synthetic disks 


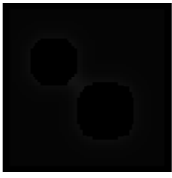

$\mathbf{P}_{x, y, 2}$

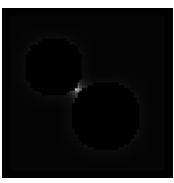

$\mathbf{P}_{x, y, 4}$

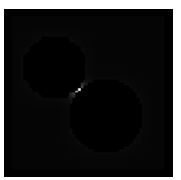

$\mathbf{P}_{x, y, 6}$

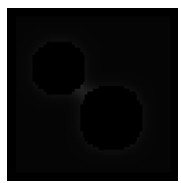

$\mathbf{P}_{x, y, 8}$

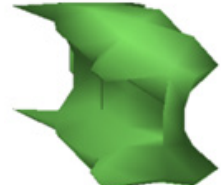

(b)

Fig. 3. (a) Nine slices of pressure domain, (b) Rendered thresholded pressure domain showing the touching surface

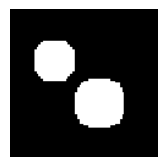

$\mathbf{S}_{x, y, 2}$

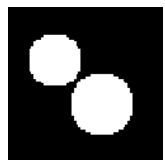

$\mathbf{S}_{x, y, 4}$

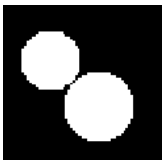

$\mathbf{S}_{x, y, 6}$

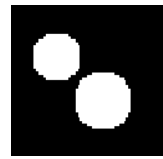

$\mathbf{S}_{x, y, 8}$

(a)

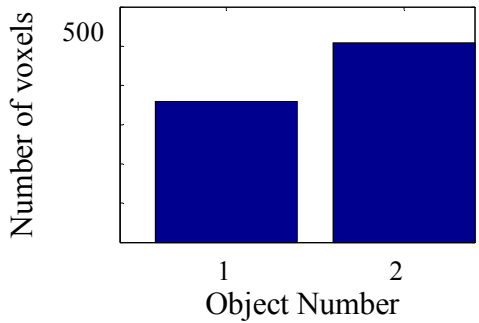

(b)

Fig. 4. (a) The two synthetic disks after applying the pressure mask, (b) Histogram of number of voxels per element after applying the pressure mask to the synthetic disks showing a detection of two objects.

For the image of Roman coins (Fig. 1) the equal density of the coins and the noise present a great challenge. The volumetric image is of resolution $444 \times 463 \times 411$ and was derived using microfocus CT. The direct application of Otsu thresholding detects only a single, large, object. Otsu thresholding was applied as a first step to the proposed method to remove the background. Additionally, a peak based threshold was applied to the intensity histogram to further separate the object from the background.

The pressure domain was calculated and thresholded to create a logical mask, Fig. 5a, which is used to discard areas with certain pressure and thus the touching regions. Finally, labelling and connectivity check performed to present the detected objects in a histogram, derived from Fig. 5b. The new histogram of objects shows that a large number of objects are detected within the set of Roman coins now that the regions where the coins touch have been excised, as expected. Objects with volume smaller than the mean volume have been removed to clearly identify significant elements among the histogram (Fig. 5c). The objects relating to these points are rendered in 


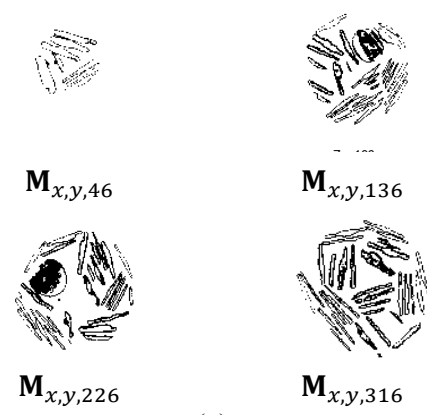

(a)

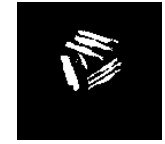

$$
\mathbf{S}_{x, y, 46}
$$

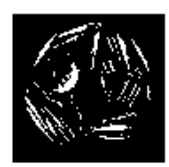

$\mathbf{S}_{x, y, 226}$

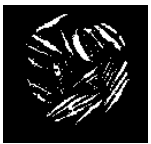

$\mathbf{S}_{x, y, 136}$

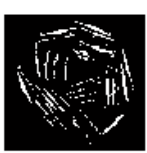

$\mathbf{S}_{x, y, 316}$

(b)

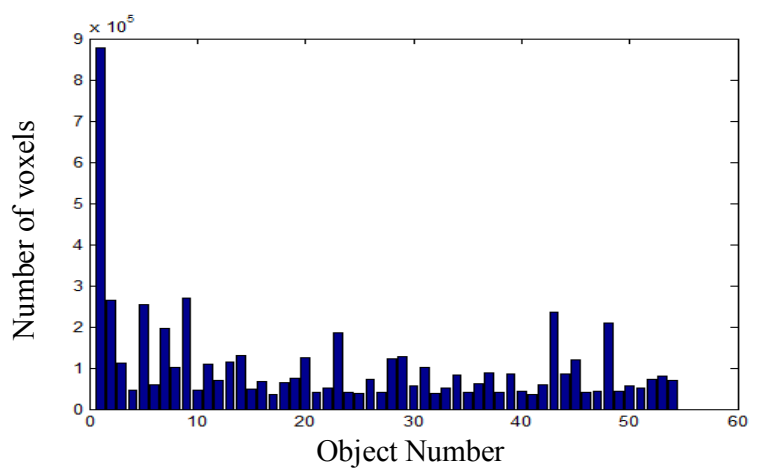

(c)

Fig. 5. (a) Pressure mask, (b) Roman coins after application of the pressure mask, (c) Histogram of the connected objects after applying the pressure mask to the roman coins and removing the mean volume

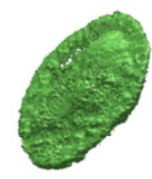

Objects 6

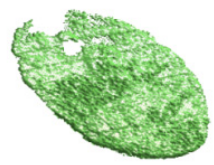

Object 30

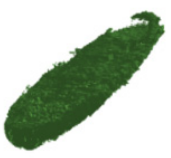

Object 10

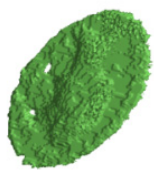

Object 42

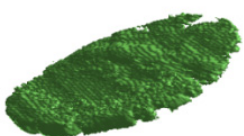

Object 16

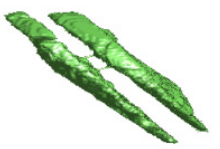

Object 45

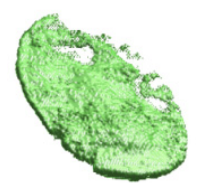

Object 22

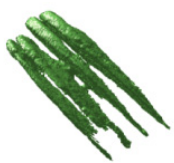

Object 48

Fig. 6. Eight roman coins extracted from the peaks in Fig. 5 (c)

(Fig. 6). This shows that the coins separation has indeed been successful and that single coins are now derived. Beyond selection of an appropriate threshold there are no other parameters associated with the new technique. 
In contrast with watershed segmentation [7], the proposed method yielded much better results. The number of detected peaks using watershed segmentation was much larger (Fig. 7a) and less significant (Fig. 7b shows a render of the main peaks in the histogram) due to the over segmentation (Fig. 7c).

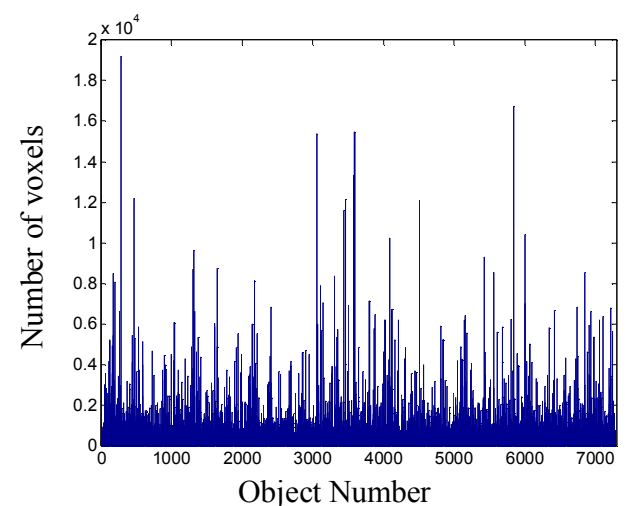

(a)

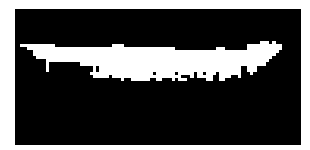

(1) Pressure Analogy Segmentation

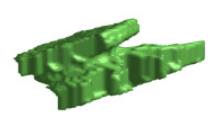

Object 290

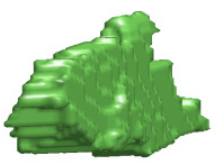

Object 4508

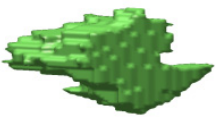

Object 3442

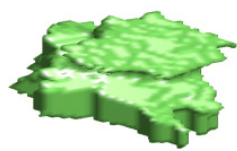

Object 6006

(b)

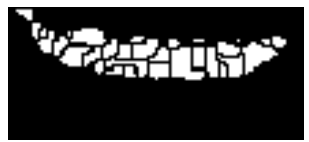

(2) Watershed Segmentation

(c)

Fig. 7. (a) Histogram of the connected objects after applying watershed to the roman coins and removing the mean volume, (b) Top peaks render, (c) (1) Segmentation by pressure analogy and (2) Over-segmentation of the same object by watershed segmentation

CT images usually suffer from two types of noise quantum noise caused by photon generation and noise introduced by the sensitivity of receptor but to furthermore study the effect of noise on the new method, Gaussian noise has been added to the image with increasing variance, Fig.8a. The number of correctly segmented objects is inversely proportional with the increase of noise (Fig. 8b) and within acceptable tolerance. The noise does naturally affect segmentation, but clearly is not catastrophic.

The main concern associated with the proposed method is the dependence on the local features and consistency in the density of the object of interest; however the application on a CT scanned orange (which has a low density and tightly connected pieces Fig.9a) also demonstrated successful segmentation results Fig. 9b and Fig 9c. 


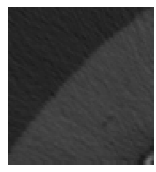

$\sigma^{2}=0$

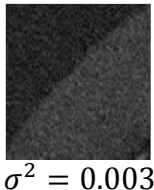

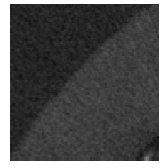

$\sigma^{2}=0.001$

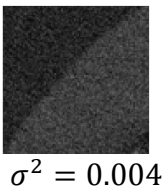

(a)

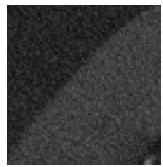

$\sigma^{2}=0.002$

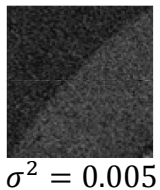

$\sigma^{2}=0.005$

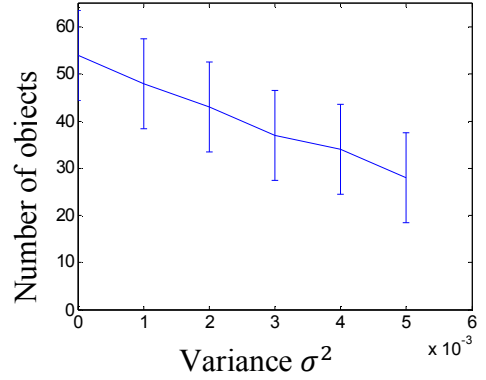

(b)

Fig. 8. (a) A crop of coins image after noise introduction with different variance values, (b) Number of objects versus the noise variance

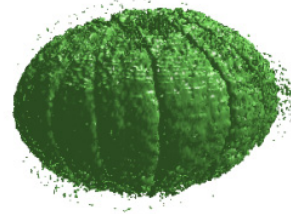

(a)

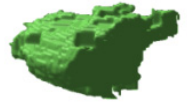

Object 3

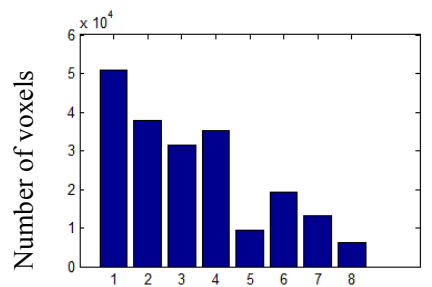

Object Number

(b)

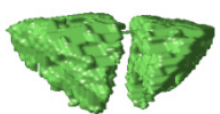

Object 4

(c)

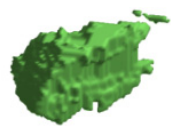

Object 7

Fig. 9. (a) Rendered CT scan of an orange, (b) Histogram of the connected objects after applying the pressure mask to the orange image, (c) Rendered objects extracted from histogram (b)

\section{Future Work}

Our analysis would be further improved by analysis of further image sources. We shall also seek to separate the complete set of coins and have the separation verified by our archaeology colleagues, and this will be part of a study on the content of the Roman hoard. This has actually been impossible until now since the only approach possible has been manual labelling of the CT image, and this is difficult and time consuming. 


\section{Conclusion}

Using the pressure domain has shown promising results for separating touching objects with the same density in CT images. The results show that it can clearly improve analysis over traditional techniques, on a selection of images. The new method is automatic, anisotropic, non-iterative and does not require a template. The results depend significantly on the local features in the original image and the threshold used for the pressure domain. One observation would be the objects with smaller touching surface area appear to have been extracted better than the others with larger areas of intersection. As such, by using the analogy of gas pressure we have a new technique which can be used to approach a known problem in (3D) image analysis.

Acknowledgments. Financial support for this study was provided by grant from the College of Technological Studies, Public Authority for Applied Education and Training, Kuwait.

\section{References}

[1] Yao, Q., Zhou, Y., Wang, J.: An Automatic Segmentation Algorithm for Touching Rice Grains Images. In: International Conference on Audio, Language and Image Processing, pp. 802-805 (November 2010)

[2] Nasr-Isfahani, S., Mirsafian, A., Masoudi-Nejad, A.: A New Approach for Touching Cells Segmentation. In: International Conference on BioMedical Engineering and Informatics, pp. 816-820 (2008)

[3] Hu, S., Hoffman, E., Reinhardt, J.M.: Automatic Lung Segmentation for Accurate Quantitation of Volumetric X-Ray CT Images. IEEE Transactions on Medical Imaging 20(6), 490-498 (2001)

[4] Bomans, M., Hohne, K.H., Tiede, U., Riemer, M.: 3-D Segmentation of MR Images of The Head For 3-D Display. IEEE Transactions on Medical Imaging 9(2), 177-183 (1990)

[5] Nixon, M.S., Liu, X.U., Direkoglu, C., Hurley, D.J.: On Using Physical Analogies for Feature and Shape Extraction in Computer Vision. The Computer Journal 54(1), 11-25 (2009)

[6] Otsu, N.: A Threshold Selection Method from Gray-Level Histograms. IEEE Transactions on Systems, Man, and Cybernetics 9(1), 62-66 (1979)

[7] Meyer, F.: Topographic Distance and Watershed Lines. Signal Processing 38(1), 113-125 (1994) 